\title{
MRD in multiple myeloma: more questions than answers?
}

\author{
Philippe Moreau ${ }^{1}$ and Elena Zamagni ${ }^{2}$
}

The growing interest in minimal residual disease (MRD) assessment in multiple myeloma (MM) is related to the high quality of responses achieved with novel agents and to the development of reliable techniques to evaluate MRD both within the bone marrow using next-generation sequencing (NGS) or next-generation flow cytometry (NGF), and outside the bone marrow using imaging techniques, such as positron emission tomographycomputed tomography (PET-CT) ${ }^{1}$. A consensus paper by the International Myeloma Working Group (IMWG), published in 2016, represents the reference document on $\mathrm{MRD}$ in $\mathrm{MM}^{2}$. However, since its publication, new data have become available, and it is of interest to discuss what other information beyond that included in the IMWG criteria should be captured in ongoing clinical trials (Table 1).

Minimal residual disease certainly matters in MM. Munshi et al. recently published a meta-analysis on 496 patients in complete response (CR), in whom an MRDnegative status was associated with a significant improvement in both progression-free survival (PFS) and overall survival $(\mathrm{OS})^{3}$. These findings were recently confirmed by the Spanish group in a pooled analysis of three PETHEMA/GEM clinical trials involving 609 patients, showing that MRD-negative status surpassed the prognostic value of CR achievement for PFS and $\mathrm{OS}^{4}$. In the paper by the Spanish group and in the majority of the trials included in the meta-analysis by Munshi et al. MRD was mostly assessed by flow cytometry, with a sensivity level of $10^{-4}$ on average. In the IMWG consensus paper, the definition of MRD negativity requires a minimum sensitivity of 1 in $10^{5}$ nucleated cells or higher both for flow and sequencing technology. The NGS technology, which is quite well standardized, routinely reaches a

\footnotetext{
Correspondence: Philippe Moreau (philippe.moreau@chu-nantes.fr)

${ }^{1}$ University Hospital, Nantes, France

${ }^{2}$ Istituto di Ematologia Seragnoli, Bologna, Italy
}

sensitivity level of $10^{-61}$. The NGF technology, may easily reach a sensitivity level of $10^{-5}$, if not $10^{-6}$, when using the standardized EuroFlow approach ${ }^{1,5}$.

Therefore, an interesting question is whether a higher level of sensitivity will result in a better predictability, and whether we should try to routinely increase the depth of MRD detection to $10^{-6}$. In the French IFM 2009 study $^{6}$, which compared RVD versus RVD plus autologous stem cell transplantation (ASCT), MRD was evaluated both by 7-color flow cytometry in all patients and by NGS where possible. Minimal residual disease negativity evaluated by flow was associated with a PFS and OS benefit (sensitivity level of $10^{-4}$ ). Of note, among flow-negative cases, the NGS technology was associated with a higher sensitivity $\left(10^{-6}\right)$ and allowed the segregation of patients into two groups: flow-negative, NGS-negative and flownegative, NGS-positive, with a significantly worse PFS outcome in the latter population ${ }^{7}$. These results indicate that $10^{-6}$ might be the ideal cut-off for the definition of MRD negativity. This is even more plausible when the number of patients reaching $10^{-6}$ in this study was 80 out of 131 evaluable patients ${ }^{7}$. A sensitivity threshold is informative and meaningful when it can be reached by a significant number of patients in a specific therapeutic strategy.

The next question is: NGS or NGF? NGS is now standardized, but the EuroFlow consortium recently described a novel NGF approach using an optimized 2-tube 8-color antibody panel for highly sensitive (close to $10^{-6}$ ) and standardized MRD detection that could be implemented in routine diagnostic procedures. In a small number of samples, a comparison of the two techniques showed a good correlation in the percentage of residual abnormal plasma cells detected, with a similar sensitivity ${ }^{5}$. In addition, the EuroFlow technology was recently evaluated in the prospective EMN02 trial, which compared ASCT to bortezomib-based conventional therapy without ASCT and showed a significant impact of flow negative MRD on 
Table 1 MRD in multiple myeloma

\begin{tabular}{|c|c|}
\hline Validated points & Open issues \\
\hline MRD negativity is a surrogate for PFS & Optimal threshold for PFS and/or OS prediction by NGS or NGF \\
\hline MRD negativity is a surrogate for OS & Need for both NGS and NGF \\
\hline MRD by NGS is standardized & Time interval to define sustained MRD negativity \\
\hline MRD by NGF (Euroflow) is standardized & Definition of loss of MRD-negative status \\
\hline MRD by NGS or NGF and PET-CT are complementary & Optimal timing for MRD assessment during and after treatment \\
\hline \multirow[t]{8}{*}{ MRD useful to compare treatment options } & Meaning of MRD negativity in specific subgroups, i.e., high-risk cytogenetics \\
\hline & Standardization of MRD by PET-CT \\
\hline & Best tracer for PET-CT \\
\hline & MRD to alter therapy: duration of maintenance, change treatment, add agents... \\
\hline & Blood-based MRD assessment \\
\hline & MRD and detection of clonal evolution \\
\hline & MRD and MGUS-like profile \\
\hline & MRD as a valid end-point for drug approval \\
\hline
\end{tabular}

$\mathrm{PFS}^{8}$. Overall, these data indicate that both techniques may be used to evaluate MRD, despite some differences in terms of applicability, availability, cost, sampling, or cell characterization.

What about the role of imaging for the assessment of MRD in 2017? New data on the role of PET-CT have recently been published. In the IFM2009 study $^{6}$, the prognostic impact of PET-CT was convincingly demonstrated ${ }^{9}$. These data were achieved in the context of a prospective study using RVD, which is one of the most effective combinations upfront, and they confirm the prognostic impact of PET-CT already described by the Little-Rock ${ }^{10}$ and Bologna ${ }^{11}$ groups. Another important piece of information provided by this study concerns the complementary role of PET-CT and flow cytometry. A subgroup of patients was evaluated by both PET-CT and by 7-color flow cytometry. Overall, the concordance between the two techniques was low. Progression-freesurvival was significantly higher for the group of patients with both a normalized PET-CT and negative MRD by flow versus patients with either PET positivity and/or MRD positivity. When using a Cox model to analyze the impact of a normalized PET-CT, negative MRD and their interaction, the only remaining factor was the interaction, indicating that these two tools may be complementary in predicting patient outcome. Indeed, although we strongly support the use of PET-CT for the evaluation of metabolic response to therapy, it is important to emphasize that both false negative and false positive results may be seen. The Little Rock group recently found that almost $10 \%$ of newly diagnosed MM patients had a false negative PET imaging at diagnosis ${ }^{12}$, indicating that new, more sensitive PET-CT tracers, or other imaging modalities, such as whole body diffusion weighted magnetic resonance imaging, should be investigated in the future. Moreover, attempts to standardize FDG PET/CT interpretation criteria are ongoing ${ }^{13}$.

In addition, other important questions remain unsolved. One relates to the concept of sustained MRD negativity. The IWMG consensus paper proposed the confirmation of NGS/NGF and PET negativity a minimum of one year apart ${ }^{2}$. This point is of utmost importance in order to define rules for stopping treatment (during maintenance for example), or to introduce the concept of cure, but, as mentioned by Kumar et al, the definition of sustained MRD negativity was arbitrarily made ${ }^{2}$. However, the number of required monitorings of MRD negativity and the time interval between them should be defined prospectively. This is also true for the new definition of relapse in the IMWG manuscript: ${ }^{2}$ 'relapse from MRD negativity, that is loss of MRD-negative status with evidence of clonal plasma cells on NGS or NGF, or positive imaging study for recurrence of MM'. What exactly is " loss of MRD-negative status $»$ : a change from $10^{-6}$ to $10^{-5}, 10^{-5}$ to $10^{-4}$ ? Do we need confirmation on two consecutive samples or is one increment sufficient to define relapse? What are the clinical implications of this finding: resumption of interrupted treatment, change of therapy, careful observation in case of absence of biochemical or clinical progression? Indeed, the definition of "loss of MRD-negative status", which needs clarification, will also impact the new definition of disease-free survival 
Table 2 Examples of ongoing academic trials evaluating MRD with NGS and/or NFG and/or PET-CT

\begin{tabular}{|c|c|}
\hline Trial & Schedule \\
\hline \multirow{5}{*}{$\begin{array}{l}\text { Cassiopeia (Intergroupe Francophone du Myélome/ } \\
\text { HOVON-NCT02541383) }\end{array}$} & Randomized trial of VTD versus VTD plus Dara (induction and consolidation) \\
\hline & All patients receive ASCT \\
\hline & Second randomization to no maintenance versus maintenance Dara \\
\hline & $\begin{array}{l}\text { MRD measurement (by NGF and NGS) will be done at baseline, post induction, post } \\
\text { consolidation and then annually }\end{array}$ \\
\hline & PET-CT at baseline and post consolidati \\
\hline \multirow[t]{5}{*}{ FORTE (GIMEMA-NCT02203643) } & $\begin{array}{l}\text { Randomized trial of CRd versus CCyd (induction } 4 \text { cycles followed by ASCT followed by } \\
\text { consolidation } 4 \text { cycles) versus CRd } 8 \text { cycles without ASCT }\end{array}$ \\
\hline & Second randomization to maintenance Lenalidomide versus maintenance \\
\hline & Lenalidomide/Carfilzomib \\
\hline & $\begin{array}{l}\text { MRD measurement (by NGF and NGS) will be done at baseline, post induction (4 } \\
\text { cycles), pre maintenance and then annually }\end{array}$ \\
\hline & PET-CT at baseline, post induction ( 4 cycles) and pre maintenance \\
\hline \multirow[t]{6}{*}{$\begin{array}{l}\text { GEM Menos } 65 \text { (PETHEMA - NCT } 01916252 \text { \& NCT } \\
\text { 02406144) }\end{array}$} & $\begin{array}{l}\text { Randomized trial of Mel200 versus Bu-Mel as conditioning regimen to ASCT following } 6 \\
\text { cycles of induction with VRD }\end{array}$ \\
\hline & All patients receive consolidation with 2 cycles of VRD \\
\hline & Second randomization to Lenalidomide-dexamethasone maintenance versus \\
\hline & Lenalidomide-dexamethasone-Ixazomib maintenance \\
\hline & $\begin{array}{l}\text { MRD measurement by NGF will be done at baseline, post induction, post ASCT, post } \\
\text { consolidation and then annually }\end{array}$ \\
\hline & $\begin{array}{l}\text { Based on NGF results following } 2 \text { years of maintenance, decision to stop maintenance } \\
\text { in case of sustained-MRD negativity, or prolong maintenance } 3 \text { additional years in case } \\
\text { of NGF positivity }\end{array}$ \\
\hline
\end{tabular}

VTD bortezomib, thalidomide, dexamethasone, ASCT autologous stem cell transplantation, Dara daratumumab, CRd carfilzomib-lenalidomide-dexamethasone, CCyd carfilzomib-cyclophosphamide-dexamethasone, VRD bortezomib-lenalidomide-dexamethasone, Mel 200 melphalan 200mg/m², Bu-Mel busulfan-melphalan, Len-dex lenalidomide-dexamethasone, Len-dex-ixa lenalidomide-dexamethasone-ixazomib, Neg negative, Pos positive

proposed in 2016, which is the duration from the start of MRD negativity to the time of reappearance of $\mathrm{MRD}^{2}$. The optimal timing for MRD assessment also remains to be defined.

Overall, MRD assessment will become key in the followup of patients with MM. Experts are in agreement that MRD negativity is one of the best prognostic markers, a surrogate for PFS and OS. It is hoped that ongoing (Table 2) and future trials will help to define the optimal use of the technologies to assess MRD, which will potentially determine and tailor our therapeutic strategies.

\section{Competing interests}

The authors declare that they have no competing interests.

Publisher's note: Springer Nature remains neutral with regard to jurisdictional claims in published maps and institutional affiliations.

Received: 30 August 2017 Revised: 6 October 2017 Accepted: 13 October 2017

Published online: 05 December 2017

\section{References}

1. Paiva, B., van Dongen, J. J. \& Orfao, A. New criteria for response assessment: role of minimal residual disease in multiple myeloma. Blood 125, 3059-3068 (2015).

2. Kumar, S. et al. International Myeloma Working Group consensus criteria for response and minimal residual disease assessment in multiple myeloma. Lancet Oncol. 17, e328-e346 (2016).

3. Munshi, N. C. et al. Association of minimal residual disease with superior survival outcomes in patients with multiple myeloma: a meta-analysis. JAMA Oncol. 3, 28-35 (2017).

4. Lahuerta, J. J. et al. Depth of response in multiple myeloma: a pooled analysis of three PETHEMA/GEM clinical trials. J. Clin. Oncol. JCO2016692517. https:// doi.org/10.1200/JCO.2016.69.2517(2017).

5. Flores-Montero, J. et al. Next Generation Flow for highly sensitive and standardized detection of minimal residual disease in multiple myeloma. Leukemia. https://doi.org/10.1038/leu.2017.29 (2017).

6. Attal, M. et al. Lenalidomide, bortezomib and dexamethasone with transplantation for myeloma. N. Engl. J. Med. 316, 1311-1320 (2017).

7. Avet-Loiseau, H. et al. Evaluation of Minimal Residual Disease by Next Generation Sequencing is highly predictive of progression-free survival in the IFM/ DFCl 2009 Trial. Blood 126, 191a (2015).

8. Oliva S. et al. Minimal residual disease by multiparameter flow cytometry in transplant eligible patients with newly diagnosed multiple myeloma: results from the EMN2/HO95 phase 3 trial. Haematologica S2, 102a (2017).

9. Moreau, P. et al. Prospective evaluation of magnetic resonance imaging and 18Fluorodeoxyglucose Positron Emission Tomography-Computed 
Tomography at diagnosis and before maintenance therapy in symptomatic patients with multiple myeloma included in the IFM/DFCI 2009 trial: results of the IMAJEM study. J. Clin. Oncol.. https://doi.org/10.1200/JCO.2017.72.2975 (2017). epub 2017 Jul 7.

10. Bartel, T. B. et al. F18-fluorodeoxyglucose positron emission tomography in the context of other imaging techniques and prognostic factors in multiple myeloma. Blood 114, 2068-2076 (2009).

11. Zamagni, E. et al. Prognostic relevance of 18-F FDG PET/CT in newly diagnosed multiple myeloma patients treated with up-front autologous transplantation. Blood 118, 5989-5995 (2011).
12. Rasche, L. et al. Low expression of hexokinase-2 is associated with falsenegative FDG-positron emission tomography in multiple myeloma. Blood $\mathbf{1 3 0}$, 30-34 (2017).

13. Nanni, C. et al. Image interpretation criteria for FDG PET/CT in multiple myeloma: a new proposal from an Italian expert panel. IMPeTUs (Italian Myeloma criteria for PET USe). Eur. J. Nucl. Med. Mol. Imaging 43, 414-421 (2016). 